\title{
Surgical approach and prognosis of primitive chest wall alveolar rhabdomyosarcoma in an adult: A case report and review of the literature
}

\author{
Narindra Njarasoa Mihaja Razafimanjato ${ }^{1}$, Tsiry Dama Ntsoa Ravelomihary ${ }^{1}$, \\ Mampionona Ranaivomanana ${ }^{2}$, Guillaume Odilon Tsiambanizafy ${ }^{1}$, Hanitrala Jean \\ Louis Rakotovao ${ }^{1}$, Francis Allen Hunald ${ }^{3}$ \\ ${ }^{1}$ DEPARTMENT OF SURGERY, THORACIC SURGERY UNIT, JOSEPH RAVOAHANGY ANDRIANAVALONA HOSPITAL, FACULTY OF MEDICINE, UNIVERSITY OF ANTANANARIVO, MADAGASCAR \\ ${ }^{2}$ DEPARTMENT OF ONCOLOGY, FACULTY OF MEDICINE, UNIVERSITY OF FIANARANTSOA, MADAGASCAR \\ ${ }^{3}$ DEPARTMENT OF SURGERY, PAEDIATRIC SURGERY UNIT, FACULTY OF MEDICINE, UNIVERSITY OF ANTANANARIVO, MADAGASCAR
}

Alveolar rhabdomyosarcomas (ARMS) are rare entities that occur predominantly on the extremities and represent $20 \%$ of rhabdomyosarcomas. Other common locations include the peri-rectal and perineal regions, head and neck, and genitourinary system. To our knowledge, this presentation is the first case of alveolar rhabdomyosarcoma of the chest wall reported in the literature. Here, we describe a case of a 64year-old man with alveolar rhabdomyosarcomas who underwent a chest wall resection. The defect was reconstructed with polypropylene mesh and latissimus dorsi muscle. Our patient did not show recurrence ten months after complete resection. The present paper describes our surgical approach to a case of primary alveolar rhabdomyosarcoma of the chest wall in an adult. The authors discuss the principal judgment criteria for successful surgical resection and prognosis, highlighting the relevance of chest wall reconstruction and their complementary aspects in the multimodal management of this rare entity.
Category: Case Presentation

Received: April 06, 2020

Accepted: July 11, 2020

Keywords:

alveolar rhabdomyosarcomas, chest wall tumour, flap, mesh reconstruction, sarcomas, thoracic surgery

*Corresponding author:

Narindra Njarasoa Mihaja Razafimanjato,

University of Antananarivo. Faculty of Medicine, HU/JRA Antananarivo, Madagascar

E-mail: razafesteban@yahoo.fr

\section{Introduction}

Soft tissue sarcomas of the chest wall represent approximately $0.1-0.15 \%$ of all adult malignancies [1,2]. It is a rare entity of the soft tissue malignancies that manifests itself as a painless, slow-growing mass [1,3]. Alveolar rhabdomyosarcomas (ARMS) are rare entities that occur predominantly in the extremities and account for $20 \%$ of rhabdomyosarcomas [4,5]. Other common locations include the peri-rectal and perineal regions, head and neck, and genitourinary system [5]. To our knowledge, our observation is the first case of alveolar rhabdomyosarcoma of the chest wall reported in the literature. Surgery is the mainstay of treatment, and represents a major challenge for surgical oncologists [1]. The common problem encountered is represented by generation of significant defects of the chest wall after resection, which requires reconstructive surgery. There is also a serious debate about the materials and techniques used to reconstruct the chest wall. Finally, there is a problem related to the cost and availability of prosthetic materials in developing countries such as ours. The present paper describes our surgical approach through a case of primary alveolar rhabdomyosarcoma of the chest wall in an adult. The authors discuss the principal judgment criteria for successful surgical resection and prognosis, highlighting the relevance of chest wall reconstruction and their complementary aspects in the multimodal management of this rare entity.

\section{Case Presentation}

We present a case of a 64-year-old man with an insignificant medical and family history, with the main complaint of a rapidly growing painless mass on the right lower thoracic base, with no history of trauma. During evaluation, the patient was afebrile but with stable vital signs. Chest examination demonstrated a large, tender, illdefined, immobile mass approximately $10 \mathrm{~cm}$ on right 
basithoracic. Imaging studies were performed, and chest computed tomography scan with contrast demonstrated a heterogeneous tissue mass $(90 \times 110 \times 70 \mathrm{~mm})$ that originated from the lower right anterolateral chest wall and protruded into the intra- and extra-pleural cavity (Figure 1).

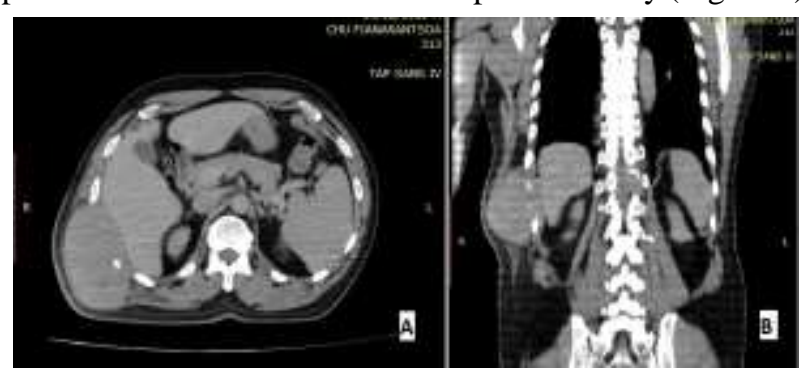

Figure 1. Preoperative CT scan demonstrates large chest wall mass on (A) axial and (B) axial views

Transcutaneous needle biopsies were successfully performed establishing a pathologic diagnosis of alveolar rhabdomyosarcomas. Patient underwent neoadjuvant chemotherapy with Adriblastine - Ifosfamide for 2 cycles. Restaging CT scan was performed finding partial remission of the tumor with a $35 \%$ decrease in tumor size without any evidence of local or distant metastases. Lytic rib lesions have not been described. The patient was referred to our unit to discuss options for resection and reconstruction. We performed wide surgical resection of the chest wall that included soft tissue resection with four segments of anterolateral ribs. The chest wall defect was reconstructed with polypropylene mesh and latissimus dorsi muscle (Figure 2).

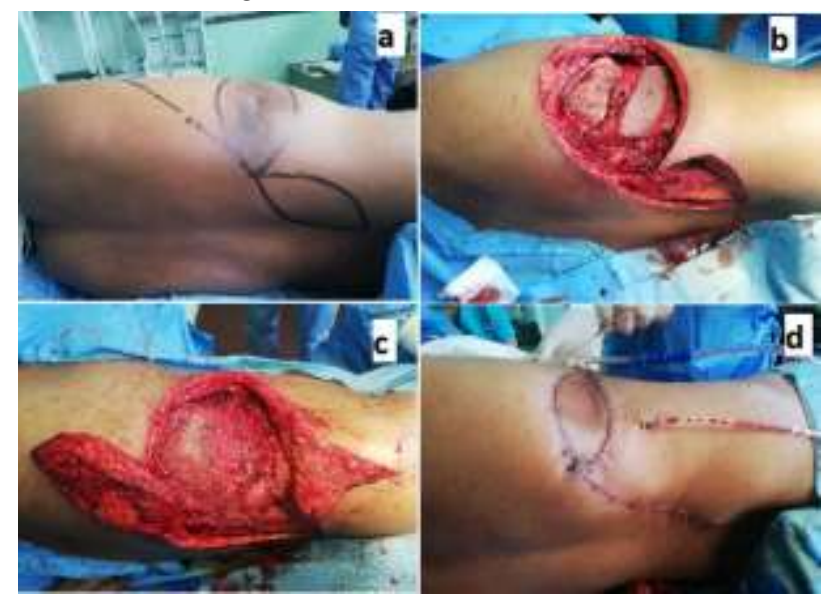

Figure 2. (a) Preoperative landmark view (b) Chest wall resection (c) Chest wall reconstruction with polypropylene mesh and latissimus dorsi muscle (d) Postoperatively view

Final pathology examination showed a tumor measuring $70 \mathrm{~cm}$ x $90 \mathrm{~cm}$ x $80 \mathrm{~cm}$, presenting $70 \%$ gross necrosis and $\mathrm{R}_{0}$ resection. The postoperative evolution was uneventful. The patient was discharged on the 15 th postoperative day. He was referred to the oncology staff for follow-up. Our patient is undergoing adjuvant chemotherapy and received radiotherapy following complete resection of tumour (50Gy over 2 months to tumor bed $+3 \mathrm{~cm}$ margins). There was no evidence of recurrence ten months after complete resection.

\section{Discussions}

Chest wall tumors have a $50 \%$ malignancy rate and comprise $<5 \%$ of all thoracic malignancies [1]. Rhabdomyosarcoma is a highly malignant skeletal muscle tumor. The alveolar form is a subtype that constitutes approximately $20 \%$ of cases which are derived from mesenchymal cells [4]. The tumor morphology is similar to alveolar structures of the lungs, so that it is named as alveolar rhabdomyosarcoma. Majority of the cases are sporadic with no genetic predisposition, which makes the prospective study of these entities to be difficult. Despite the constant development of modern surgical techniques, the surgical resection remains (since the 1960s and 1970s) the mainstay of therapeutic management of early stage of the chest-wall sarcomas [1,6]. Even if recent studies demonstrate the effectiveness of chemotherapy and radiation, surgery would be more useful for tumor resection, not only securing the negative surgical margins but also reconstructing chest wall defects in large tumors.

\section{Oncological surgical approach of chest wall rhabdomyosarcomas}

In a comprehensive review of the literature, the treatment of patients with localized sarcoma was generally represented by complete surgical resection, an approach that seems to be in fact the gold standard of the literature [7]. In most cases the aim of surgical resection is curative (with microscopically negative margins to improve local control and avoid local recurrences), although palliative resection is sometimes necessary in cases of metastatic disease [8]. Daniel J Weber et al [9] report a palliative resection of a chest wall sarcoma for refractory hemorrhage, with an uneventful recovery and no local recurrence for 8 months post-operatively. In practice, there is no rational evidence in soft tissue sarcoma surgery, in which marginal resections appear to be sufficient to control the local disease [8]. In previous studies, surgery with a wide margin is a safe and good technique for the treatment of primary chest wall tumours with acceptable morbidity and mortality [7]. In theory, the resection should remove all the tumor cells that could potentially lead to local recurrences or metastases. The size of the surgical margin is usually 1 to $2 \mathrm{~cm}$ wide, to ensure there are no malignancies cells found around the tumor (called a negative or clean margin). Pisters PW et al. published in 1996 the largest series of 1,041 localized soft tissue sarcomas, and were able to demonstrate this theory by establishing an association between microscopically positive surgical margin and tumor-related mortality [10]. However, recent publications have shown that margins are 
an important and independent factor in the local control and metastasis prevention, the negative surgical margins being not significantly associated with better overall survival than the positive margins [8]. There has thus been a paradigm shift in the limits of surgical resection, from large radical resections to narrower marginal resections [8,11]. This dogma has been debated by several authors in the literature. In 2004, McKee et al. conducted a quantitative evaluation of negative-margin resection in 111 patients after tumor resection [12]. In their multivariate analysis, negative margins $\geq 10 \mathrm{~mm}$ were associated with better local control than $<10 \mathrm{~mm}$ borders. However, larger resection margins did not increase overall patient survival. They conclude that a safety margin of $\geq 10 \mathrm{~mm}$ on histological examination minimizes the risk of local recurrence. A similar conclusion was established by Dickinson et al in a retrospective analysis of 279 patients with localized soft tissue sarcoma, and concludes that surgical resection with a negative margin width $>1 \mathrm{~mm}$ is sufficient to achieve the successful outcomes [13]. Conversely, Ahmad et al. obtained in 2016, on 235 patients classified by negative margin widths such as $\leq 1 \mathrm{~mm}, 1-5 \mathrm{~mm}$, and $>5 \mathrm{~mm}$, the same result for local control and survival [14]. These studies showed that the negative margin width obtained did not affect local control, disease-specific survival or metastatic-free survival, whether or not adjuvant radiation was applied. Based on these retrospective results, the net margins may be similar as long as the tumor is resected $\mathrm{R} 0$. In a retrospective study of overall survival (OS), local recurrence-free survival (LRFS) and disease-free survival (DFS) carried by Van Geel AN et al and analyzing twenty-one prognostic factors for survival in sixty consecutive patients, the most important factors that determine the prognosis of patients who undergo chest wall surgery for sarcoma or chondrosarcoma include histologic grade, presence or absence of metastatic disease, and complete resection [15]. Predictors of recurrence have been described as a highgrade tumor on pathology, tumor size $>5 \mathrm{~cm}$ and high uptake on PET/CT [2]. The five-year survival rate for wide local excision of primary sarcoma was $63 \%$ according to the study published by the Department of Surgical Oncology and Medical Statistics Netherlands Cancer Institute [7]. Neoadjuvant chemotherapy indicated for locally advanced sarcomas with high histological risk has several advantages but has also been widely discussed in the literature. Firstly, it can allow for easier resection of the tumor and improve the possibilities of conservative surgery during preoperative administration. A similar approach is applied in our case, which resulted in a successful recovery. Another potential advantage of neoadjuvant chemotherapy is the increased success in achieving negative histological margins, which are associated with a reduced risk of local recurrence and improved survival. Besides, the preoperative tumor response obtained after neoadjuvant chemotherapy is useful to the medical oncologist in the selection of drugs for adjuvant chemotherapy; in addition, when neoadjuvant chemotherapy is administered concurrently with radiotherapy (neoadjuvant chemoradiation) it acts as a radiosensitizer $[3,16,17]$.

Table I. Overview of indications and options for reconstruction

\begin{tabular}{|c|c|c|}
\hline & Reconstruction of thoracic skeleton & Reconstruction of thoracic soft tissues \\
\hline Indications & \multicolumn{2}{|c|}{$\begin{array}{l}\text { Defect affects more than resection more than } 3-4 \text { ribs or is size } \geq 5 \mathrm{~cm} \text { (stabilize paradoxical } \\
\text { motion and protect vital organs) } \\
\text { May reconstruct smaller defect if underlying pulmonary disease } \\
\text { May not reconstruct larger defects if previous radiotherapy } \\
\text { If defect is located posteriorly and/or superiorly (shielded by the scapula), then a larger defect is } \\
\text { tolerated. }\end{array}$} \\
\hline Options & $\begin{array}{l}\text { Alloplastic reconstruction: } \\
\text { Mesh } \\
\text { Methylmethacrylate } \\
\text { Bone grafts } \\
\text { A cellular dermal matrix } \\
\text { Titanium plate fixation }\end{array}$ & $\begin{array}{l}\text { Autogenous tissue reconstruction: } \\
\text { Anterior chest wall and mediastinum } \\
\text { - Pectoralis Major } \\
\text { - Latissmus Dorsi } \\
\text { - Omentum } \\
\text { - Rectus Abdominis } \\
\text { Anterolateral chest wall } \\
\text { - Latissmus Dorsi } \\
\text { - Omentum } \\
\text { - Rectus Abdominis } \\
\text { - External Oblique } \\
\text { - Serratus Anterior } \\
\text { - Transverse thoracoabdominal flap }\end{array}$ \\
\hline
\end{tabular}


2. Aims, methods and indications of chest wall reconstruction (Table I).

The proper oncologic resection often presents a problem in the reconstruction of the chest wall, depending on the extent of the malignant tumor and requiring reconstruction after tumor resection. The aims of chest wall reconstruction are (a) erase dead space, (b) provide tissue coverage to protect vital organs, (c) improve chest wall stability to restore the biomechanics of the chest wall and (d) ensure a satisfactory aesthetic result. The indication for reconstructive surgery is determined by nature, size $(>5$ $\mathrm{cm}$ ) and topography of the anterolateral defect (high lateral resection of more than 3-4 ribs), as well as the patient's general health and prognosis [18]. An ideal material and method of chest wall reconstruction should be based according to literature on the following criteria [19]: (a) High flexibility, rigidity and stability to avoid postoperative paradoxical respiration, (b) No rejection/ reaction of transplant and less risk of infection, (c) radiotransparency for more accurate radiological follow-up of eventual local recurrence. The regional pedicled muscular or musculocutaneous flaps are the first choice for soft tissue coverage of chest wall defects. Flaps such as latissimus dorsi, vertical rectus abdominis (VRAM) or transverse rectus abdominis (TRAM), and external pectoral, serratus and oblique muscles are commonly used. Also, many local and pedicular flaps can be used in combination $[1,6]$. In our case, the latissimus dorsi flap is used for tissue covering in our case. The advantage of using the musculocutaneous flap of the great dorsal fin is twofold, due to its size and its large rotation angle which permits it to be transferred at a distance and sufficiently cover most of the chest wall defects, up to correct the intrathoracic dead spaces [6,8]. On the other hand, its dominant vascular pedicle (having a diameter of 2 to $4 \mathrm{~mm}$ ) is reliable and allows immediate vascular microsurgery, if necessary [20]. Other composite implant techniques such as polypropylene mesh or MARLEX ${ }^{\circledR}$ mesh, polytetrafluoroethylene (GORETEX $\AA$ ), silicone, rubber, carbon, have been described in case reports [19]. Reports have also been written on the safe use of titanium implants in chest wall reconstruction after tumor resection with cases of infection and major complication such as acute respiratory failure [18]. Several authors have recommended the use of a combination of polypropylene mesh and methyl methacrylate substitutes in the form of a "sandwich" prosthesis to maintain chest wall stiffness and to improve functional and aesthetic results after large chest wall resections [6,8]. However, Michael J. Weyant et al. carried out a comparative study of results of chest wall reconstruction with and without rigid prosthesis in 262 patients underwent chest wall resection at Memorial SloanKettering Cancer Center [21]. After 90 days of reconstruction, the occurrence of postoperative complications of the defects reconstructed with MMM
(Marlex mesh-Methyl-Methacrylate sandwich) is significantly higher than chest wall reconstruction with non-rigid methods (PTFE: polytetrafluoroethylene or PPM: polypropylene mesh) with $\mathrm{p}$-value $<0.006$. The impermeability of methyl methacrylate substitutes to fluids increases the risk of infectious complications of this technique [8]. In our center, our first choice in practice is non-absorbable polypropylene mesh in parietal reconstructions and hernia repairs because of its inert, radiolucent, resistant and sufficiently flexible properties. Each material has its benefits and limitations. In literature, there is still a lack of evidence for each of these approaches, and additional studies are justified to provide long-term results. In our view, the choice of implant quality and use generally depends on three criteria including its availability, the local infectious status and the surgeon's expertise.

\section{Conclusions}

As more cases are recognized and reported, we hope to be able to better define the value of reconstructive surgery in this rare entity. A multidisciplinary approach including thoracic surgery, plastic surgery, medical oncology, and radiation oncology is crucial to optimal management of this difficult disease process. A thoracic surgeon's practical knowledge of reconstructive surgery is an imperative for the management of this pathology in a developing country such as Madagascar.

\section{Conflict of interest disclosure}

There are no known conflicts of interest in the publication of this article. This research received no specific grant from any funding agency in the public, commercial, or not-for-profit sectors. The manuscript was read and approved by all authors.

\section{Compliance with ethical standards}

Any aspect of the work covered in this manuscript has been conducted with the ethical approval of all relevant bodies and that such approvals are acknowledged within the manuscript. The authors declare that the involved patient gave his informed consent for participation in research. The study was done according to the declaration of Helsinki.

\section{Authors contributions}

RNNM and RM were a major contributor in drafting the manuscript and revised the content.

RNNM, TOG and RTDN, were the surgeon responsible for treating the patient.

RHJL and HFA revised the manuscript for important content. 


\section{References}

1. Davis CH, Yammine H, Khaitan PG, Chan EY, Kim MP. A multidisciplinary approach to giant soft tissue sarcoma of the chest wall: A case report. Int J Surg Case Rep. 2016;28:211-213. doi:10.1016/j.ijscr.2016.10.002

2. Kress RL, Dalwadi SM, Irani AD. R0 resection and reconstruction for a large, rapidly progressive chest wall sarcoma. J Cardiothorac Surg. 2018;13(1):127. doi:10.1186/s13019-018-0812-8

3. Czarny MJ, Chow GV, Rhee DS, et al. Sarcoma of the chest wall: a rare tumor. Am J Med. 2010;123(2):e7-e8. doi:10.1016/j.amjmed.2009.07.030

4. Kandola SK, Rai MP, Rao SL, Marinas EB. Alveolar rhabdomyosarcoma presenting as a lung mass: an uncommon presentation of a less common tumor. BMJ Case Rep. 2018;2018:bcr2018224986. Published 2018 Mar 27. doi:10.1136/bcr-2018-224986

5. Corneal J, Cordell C, Geli D. et al. Alveolar Rhabdomyosarcoma: An Unusual Presentation. Am J Clin Pathol. 2012;138(suppl_2):A017.

6. Tukiainen E. Chest wall reconstruction after oncological resections. Scand J Surg. 2013;102(1):913. doi:10.1177/145749691310200103

7. Akhtar A, Shah S, Sheikh AB, et al. Sarcoma Arising from the Chest Wall: A Case Report. Cureus. 2017;9(8):e1604. doi:10.7759/cureus.1604

8. Harati K, Kolbenschlag J, Behr B, et al. Thoracic Wall Reconstruction after Tumor Resection. Front Oncol. 2015;5:247. doi:10.3389/fonc.2015.00247

9. Weber DJ, Coleman JJ, Kesler KA. Refractory bleeding from a chest wall sarcoma: a rare indication for palliative resection. $J$ Cardiothorac Surg. 2013;8:82. doi:10.1186/1749-8090-8-82

10. Pisters PW, Leung DH, Woodruff J, Shi W, Brennan MF. Analysis of prognostic factors in 1,041 patients with localized soft tissue sarcomas of the extremities. $J$ Clin Oncol. 1996;14(5):1679-1689. doi:10.1200/JCO.1996.14.5.1679

11. Harati K, Lehnhardt M. The changing paradigm of resection margins in sarcoma resection. Innov Surg Sci. 2017;2(4):165-170. doi:10.1515/iss-2017-0043
12. McKee MD, Liu DF, Brooks JJ, Gibbs JF, Driscoll DL, Kraybill WG. The prognostic significance of margin width for extremity and trunk sarcoma. J Surg Oncol. 2004;85(2):68-76. doi:10.1002/jso.20009

13. Dickinson IC, Whitwell DJ, Battistuta D, et al. Surgical margin and its influence on survival in soft tissue sarcoma. ANZ J Surg. 2006;76(3):104-109. doi: 10.1111/j.1445-2197.2006.03615.x

14. Ahmad R, Jacobson A, Hornicek F, et al. The Width of the Surgical Margin Does Not Influence Outcomes in Extremity and Truncal Soft Tissue Sarcoma Treated With Radiotherapy. Oncologist. 2016;21(10):12691276. doi:10.1634/theoncologist.2015-0534

15. van Geel AN, Wouters MW, Lans TE, Schmitz PI, Verhoef C. Chest wall resection for adult soft tissue sarcomas and chondrosarcomas: analysis of prognostic factors. World J Surg. 2011;35(1):63-69. doi: 10.1007/s00268-010-0804-x

16. Pasquali S, Gronchi A. Neoadjuvant chemotherapy in soft tissue sarcomas: latest evidence and clinical implications. Ther Adv Med Oncol. 2017;9(6):415-429. doi:10.1177/1758834017705588

17. Flugstad DL, Wilke CP, McNutt MA, Welk RA, Hart MJ, McQuinn WC. Importance of surgical resection in the successful management of soft tissue sarcoma. Arch Surg. 1999;134(8):856-862. doi:10.1001/archsurg.134.8.856

18. Gao E, Li Y, Zhao T, et al. Reconstruction of anterior chest wall: a clinical analysis. J Cardiothorac Surg. 2018;13(1):124. doi:10.1186/s13019-018-0810-x

19. Sanna S, Brandolini J, Pardolesi A, et al. Materials and techniques in chest wall reconstruction: a review. $J$ Vis Surg. 2017;3:95. doi:10.21037/jovs.2017.06.10

20. Sauerbier M, Dittler S, Kreutzer C. Microsurgical chest wall reconstruction after oncologic resections. Semin Plast Surg. 2011;25(1):60-69. doi:10.1055/s-00311275172

21. Weyant MJ, Bains MS, Venkatraman E, et al. Results of chest wall resection and reconstruction with and without rigid prosthesis. Ann Thorac Surg. 2006;81(1):279-285.

doi:10.1016/j.athoracsur.2005.07.001 